\title{
Existence of Positive Weak Solutions for a New Class of $(p, q)$ Laplacian Nonlinear Elliptic System with Sign-Changing Weights
}

\author{
Rafik Guefaifia $\mathbb{D}^{1},{ }^{1}$ Salah Mahmoud Boulaaras $\mathbb{D}^{2,3}$ Sultan Alodhaibi, ${ }^{2}$ \\ and Salem Alkhalaf $\mathbb{i D}^{4}$ \\ ${ }^{1}$ Department of Mathematics, Faculty of Exact Sciences, Larbi Tebessi University, Tebessa 12002, Algeria \\ ${ }^{2}$ Department of Mathematics, College of Sciences and Arts, Al-Rass, Qassim University, Saudi Arabia \\ ${ }^{3}$ Laboratory of Fundamental and Applied Mathematics of Oran (LMFAO), University of Oran 1, Ahmed Benbella, Algeria \\ ${ }^{4}$ Department of Computer, College of Sciences and Arts, Al-Rass, Qassim University, Saudi Arabia
}

Correspondence should be addressed to Salah Mahmoud Boulaaras; s.boularas@qu.edu.sa

Received 10 February 2020; Revised 9 April 2020; Accepted 18 April 2020; Published 4 May 2020

Guest Editor: Chun-Lai Li

Copyright (c) 2020 Rafik Guefaifia et al. This is an open access article distributed under the Creative Commons Attribution License, which permits unrestricted use, distribution, and reproduction in any medium, provided the original work is properly cited.

In this paper, by using subsuper solutions method, we study the existence of weak positive solutions for a new class of $(p, q)$ Laplacian nonlinear elliptic system in bounded domains, when $a(x), b(x), \alpha(x)$, and $\beta(x)$ are sign-changing functions that maybe negative near the boundary, without assuming sign conditions on $f(0), g(0), h(0)$, and $\gamma(0)$.

\section{Introduction}

The study of differential equations and variational problems with nonstandard $p(x)$-growth conditions is a new and interesting topic. It arises from nonlinear elasticity theory, electrorheological fluids, etc. (see [1-15]). Many existence results have been obtained on this kind of problems, see, for example [10,12,16-18] and [19-30]. In $[31,32]$, Fan et al. studied the regularity of solutions for differential equations with nonstandard $p(x)$--growth conditions.

In this article, we consider the following system:

$$
\left\{\begin{array}{l}
-\triangle_{p} u-|u|^{p-2} u=\lambda_{1} a(x) f(v)+\mu_{1} \alpha(x) h(u) \text { in } \Omega \\
-\triangle_{q} v-|v|^{q-2} v=\lambda_{2} b(x) g(u)+\mu_{2} \beta(x) \gamma(v) \text { in } \Omega \\
u=v=0 \text { on } \partial \Omega
\end{array}\right.
$$

where $\Delta_{s} z=\operatorname{div}\left(|\nabla z|^{s-2} \nabla z\right), \quad s>1, \Omega \subset \mathbb{R}^{N}(N \geq 3)$ is a bounded domain with smooth boundary $\partial \Omega, a(x), b(x)$, $\alpha(x), \beta(x) \in C(\bar{\Omega})$, and $\lambda_{1}, \lambda_{2}, \mu_{1}$, and $\mu_{2}$ are nonnegative parameters.
In fact, we study the existence of positive solutions to system (1) with sign-changing weight functions $a(x), b(x)$, $\alpha(x)$, and $\beta(x)$. Due to these weight functions, the extensions are challenging and nontrivial.

These problems arise in some physical models and are interesting in applications at combustion, mathematical biology, and chemical reactions. Our approach is based on the method of sub- and supersolutions (see $[6,23-25,27,30])$.

We make the following assumptions:

$(H 1) f, g, h, \gamma \in C^{1}([0, \infty))$ are nondecreasing functions such that $\lim _{s \longrightarrow+\infty} f(s)=\lim _{s \longrightarrow+\infty}$
$g(s)=\lim _{s \longrightarrow+\infty} h(s)=\lim _{s \longrightarrow+\infty} \gamma(s)=+\infty$

$$
(H 2) \lim _{s \longrightarrow+\infty}\left(\left(f\left(M(g(s))^{(1 / q-1)}\right)\right) / s^{p-1}\right)=0, \forall M>0
$$$$
(H 3) \lim _{s \longrightarrow+\infty}\left(h(s) / s^{p-1}\right)=\lim _{s \longrightarrow+\infty}\left(\gamma(s) / s^{q-1}\right)=0
$$

Let $\sigma_{r}$ be the first eigenvalue of $-\Delta_{r}$ with Dirichlet boundary conditions and $\phi_{r}$ be the corresponding eigenfunction with $\phi_{r}>0$ in $\Omega$ and $\left\|\phi_{r}\right\|=1$ for $r=p$, q. Let $m, \eta, \delta>0$ be such that $\left(\left|\nabla \phi_{r}\right|^{r}-\sigma_{r} \phi_{r} \geq m\right)$ on 
$\overline{\Omega_{\delta}}=\{x \in \Omega, d(x, \partial \Omega) \leq \delta\}$ and $\phi_{r} \geq \eta$ on $\left(\Omega / \bar{\Omega}_{\delta}\right)$ for $r=p, q$.

Here, we assume that the weights $a(x), b(x), \alpha(x)$, and $\beta(x)$ take negative values in $\overline{\Omega_{\delta}}$ but require $a(x), b(x), \alpha(x)$, and $\beta(x)$ to be strictly positive in $\left(\Omega / \bar{\Omega}_{\delta}\right)$. To be precise, we assume that there exist positive constants $a_{0}, a_{1}, b_{0}, b_{1}, \alpha_{0}$, $\alpha_{1}, \beta_{0}$, and $\beta_{1}$ such that

$$
\begin{aligned}
a(x) & \geq-a_{0}, \\
b(x) & \geq-b_{0}, \\
\alpha(x) & \geq-\alpha_{0}, \\
\beta(x) & \geq-\beta_{0}, \\
x & \in \overline{\Omega_{\delta}}, \\
a(x) & \geq a_{1}, \\
b(x) & \geq b_{1}, \\
\alpha(x) & \geq \alpha_{1}, \\
\beta(x) & \geq \beta_{1}, \\
x & \in\left(\Omega / \bar{\Omega}_{\delta}\right) .
\end{aligned}
$$
and

Also, let $s_{0} \geq 0$ be such that $f\left(s_{0}\right), g\left(s_{0}\right), h\left(s_{0}\right), \gamma\left(s_{0}\right)>0$,

$$
\begin{aligned}
K_{1} & =\left(\frac{p-1}{p}\right) \eta^{p /(p-1)}, \\
K_{2} & =\left(\frac{q-1}{q}\right) \eta^{q /(q-1)}, \\
\theta_{0} & =\max \left\{\left(\frac{s_{0}}{K_{1}}\right)^{1 /(p-1)},\left(\frac{s_{0}}{K_{2}}\right)^{q /(q-1)}\right\} .
\end{aligned}
$$

For $\theta>\theta_{0}$, we define

$$
\begin{gathered}
\int_{\Omega}|\nabla u|^{p-2} \nabla u \cdot \nabla \xi \mathrm{d} x-\int_{\Omega}|u|^{p-2} u \cdot \xi \mathrm{d} x=\lambda_{1} \int_{\Omega} a(x) f(v) \xi \mathrm{d} x+\mu_{1} \int_{\Omega} \alpha(x) h(u) \xi \mathrm{d} x \text { in } \Omega, \\
\int_{\Omega}|\nabla v|^{q-2} \nabla v \cdot \nabla \zeta \mathrm{d} x-\int_{\Omega}|v|^{q-2} v \cdot \zeta \mathrm{d} x=\lambda_{2} \int_{\Omega} b(x) g(u) \zeta \mathrm{d} x+\mu_{2} \int_{\Omega} \beta(x) \gamma(v) \zeta \mathrm{d} x \operatorname{in} \Omega,
\end{gathered}
$$

We give the following two definitions before we give our Definition 1. Let $(u, v) \in W^{1, p}(\Omega) \cap C(\bar{\Omega}) \times W^{1, q}(\Omega) \cap$ $C(\bar{\Omega}),(u, v)$ be said a weak solution of (1) if it satisfies subsolution and supersolution of (1) if they satisfy $(\underline{u}, \underline{v}),(\bar{u}, \bar{v})=(0,0)$ on $\partial \Omega$ :

Definition 2. A pair of nonnegative functions $(\underline{u}, \underline{v}),(\bar{u}, \bar{v})$ in $W^{1, p}(\Omega) \cap C(\bar{\Omega}) \times W^{1, q}(\Omega) \cap C(\bar{\Omega}) \quad$ is called a weak

$$
\begin{gathered}
\int_{\Omega}|\nabla \underline{u}|^{p-2} \nabla \underline{u} \cdot \nabla \xi \mathrm{d} x-\int_{\Omega}|\underline{u}|^{p-2} \underline{u} \cdot \xi \mathrm{d} x \leq \lambda_{1} \int_{\Omega} a(x) f(\underline{v}) \xi \mathrm{d} x+\mu_{1} \int_{\Omega} \alpha(x) h(\underline{u}) \xi \mathrm{d} x \text { in } \Omega, \\
\int_{\Omega}|\nabla \underline{v}|^{q-2} \nabla \underline{v} \cdot \nabla \zeta \mathrm{d} x-\int_{\Omega}|\underline{v}|^{q-2} \underline{v} \cdot \zeta \mathrm{d} x \leq \lambda_{2} \int_{\Omega} b(x) g(\underline{u}) \zeta \mathrm{d} x+\mu_{2} \int_{\Omega} \beta(x) \gamma(\underline{v}) \zeta \mathrm{d} x \text { in } \Omega, \\
\int_{\Omega}|\nabla \bar{u}|^{p-2} \nabla \bar{u} \cdot \nabla \xi \mathrm{d} x-\int_{\Omega}|\bar{u}|^{p-2} \bar{u} \cdot \xi \mathrm{d} x \geq \lambda_{1} \int_{\Omega} a(x) f(\underline{v}) \xi \mathrm{d} x+\mu_{1} \int_{\Omega} \alpha(x) h(\bar{u}) \xi \mathrm{d} x \text { in } \Omega, \\
\int_{\Omega}|\nabla \bar{v}|^{q-2} \nabla \bar{v} \cdot \nabla \zeta \mathrm{d} x-\int_{\Omega}|\bar{v}|^{q-2} \bar{v} \cdot \zeta \mathrm{d} x \geq \lambda_{2} \int_{\Omega} b(x) g(\bar{u}) \zeta \mathrm{d} x+\mu_{2} \int_{\Omega} \beta(x) \gamma(\bar{v}) \zeta \mathrm{d} x \text { in } \Omega,
\end{gathered}
$$


for all $(\xi, \zeta) \in W_{0}^{1, p}(\Omega) \times W_{0}^{1, q}(\Omega)$.

We shall establish the following result.

Theorem 1. Assume that the conditions $(H 1)-(H 3)$ hold, $a(x), b(x), \alpha(x)$, and $\beta(x)$ are in $L^{\infty}(\Omega)$ and $\Lambda \neq \varnothing$. Let $I=\cup_{\theta \in \Lambda}\left[\lambda_{1 *}(\theta), \lambda_{1}^{*}(\theta)\right] \times\left[\mu_{1 *}(\theta), \mu_{1}^{*}(\theta)\right] \times\left[\lambda_{2 *}(\theta), \lambda_{2}^{*}(\theta)\right]$ $\times\left[\mu_{2 *}(\theta), \mu_{2}^{*}(\theta)\right]$.

Then, problem (1) has a positive weak solution for each $\left(\lambda_{1}, \mu_{1}, \lambda_{2}, \mu_{2}\right) \in I$.
Proof of Theorem 1. Let $\left(\lambda_{1}, \mu_{1}, \lambda_{2}, \mu_{2}\right) \in I$ and $\theta>\theta_{0}$ be such that $\quad\left(\lambda_{1}, \mu_{1}, \lambda_{2}, \mu_{2}\right) \in\left[\lambda_{1 *}(\theta), \quad \lambda_{1}^{*}(\theta)\right] \times\left[\mu_{1 *}(\theta), \mu_{1}^{*}\right.$ $(\theta)] \times\left[\lambda_{2 *}(\theta), \lambda_{2}^{*}(\theta)\right] \times\left[\mu_{2 *}(\theta), \mu_{2}^{*}(\theta)\right]$.

We shall verify that

$$
\begin{aligned}
& \underline{u}=\theta^{1 /(p-1)}\left(\frac{p-1}{p}\right) \phi_{p}^{p /(p-1)}, \\
& \underline{v}=\theta^{1 /(q-1)}\left(\frac{q-1}{q}\right) \phi_{q}^{q /(q-1)},
\end{aligned}
$$

is a subsolution of (1). Let the test function $\xi(x) \in W_{0}^{1, p}(\Omega)$ with $\xi(x) \geq 0$. We have

$$
\begin{aligned}
\int_{\Omega}|\nabla \underline{u}|^{p-2} \nabla \underline{u} \cdot \nabla \xi \mathrm{d} x-\int_{\Omega}|\underline{u}|^{p-2} \underline{u} \cdot \xi \mathrm{d} x & \leq \int_{\Omega}|\nabla \underline{u}|^{p-2} \nabla \underline{u} \cdot \nabla \xi \mathrm{d} x \\
& =\theta \int_{\Omega} \phi_{p}\left|\nabla \phi_{p}\right|^{p-2} \nabla \phi_{p} \cdot \nabla \xi \mathrm{d} x \\
& =\theta \int_{\Omega}\left[\left|\nabla \phi_{p}\right|^{p-2} \nabla \phi_{p} \cdot \nabla\left(\phi_{p} \xi\right)-\left|\nabla \phi_{p}\right|^{p} \xi\right] \mathrm{d} x \\
& =\theta \int_{\Omega}\left(\sigma_{p} \phi_{p}^{p}-\left|\nabla \phi_{p}\right|^{p}\right) \xi \mathrm{d} x .
\end{aligned}
$$

Similarly,

$\int_{\Omega}|\nabla \underline{v}|^{q-2} \nabla \underline{v} \cdot \nabla \zeta \mathrm{d} x-\int_{\Omega}|\underline{v}|^{q-2} \underline{v} \cdot \zeta \mathrm{d} x \leq \theta \int_{\Omega}\left(\sigma_{q} \phi_{q}^{q}-\left|\nabla \phi_{q}\right|^{q}\right) \zeta \mathrm{d} x$.
For all $\zeta(x) \in W_{0}^{1, q}(\Omega)$ with $\zeta(x) \geq 0$.

Now, on $\overline{\Omega_{\delta}}$ we have

$$
\begin{aligned}
\theta \int_{\overline{\Omega_{\delta}}}\left(\sigma_{p} \phi_{p}^{p}-\left|\nabla \phi_{p}\right|^{p}\right) \xi \mathrm{d} x & \leq-\theta m \int_{\overline{\Omega_{\delta}}} \xi \mathrm{d} x \\
& =-\theta m\left(\frac{1}{d}+\frac{1 d}{d^{\prime}}\right) \int_{\overline{\Omega_{\delta}}} \xi \mathrm{d} x \\
& \leq\left[-\lambda_{1} a_{0} f\left(\theta^{(1 / q-1)}\right)-\mu_{1} \alpha_{0} h\left(\theta^{(1 / p-1)}\right)\right] \int_{\overline{\Omega_{\delta}}} \xi \mathrm{d} x \\
& \leq \int_{\overline{\Omega_{\delta}}}\left[-\lambda_{1} a_{0} f\left(\theta^{(1 / q-1)}\left(\frac{q-1}{q}\right) \phi_{q}^{q / q-1}\right)-\mu_{1} \alpha_{0} h\left(\theta^{(1 / p-1)}\left(\frac{p-1}{p}\right) \phi_{p}^{p / p-1}\right)\right] \xi \mathrm{d} x \\
& \leq \int_{\overline{\Omega_{\delta}}}\left[\lambda_{1} a(x) f(\underline{v})+\mu_{1} \alpha(x) h(\underline{u})\right] \xi \mathrm{d} x \\
\theta \int_{\overline{\Omega_{\delta}}}\left(\sigma_{q} \phi_{q}^{q}-\left|\nabla \phi_{q}\right|^{q}\right) \zeta \mathrm{d} x & \leq-\theta m \int_{\overline{\Omega_{\delta}}} \zeta \mathrm{d} x, \\
& =\left(\frac{-\theta m}{d}-\frac{\theta m}{d^{\prime}}\right) \int_{\overline{\Omega_{\delta}}} \zeta \mathrm{d} x \\
& \leq\left[-\lambda_{2} b_{0} g\left(\theta^{(1 / p-1)}\right)-\mu_{2} \beta_{0} \gamma\left(\theta^{(1 / q-1)}\right)\right] \int_{\overline{\Omega_{\delta}}} \zeta \mathrm{d} x \\
& \leq \int_{\overline{\Omega_{\delta}}}\left[-\lambda_{2} b_{0} g\left(\theta^{(1 / p-1)}\left(\frac{p-1}{p}\right) \phi_{p}^{p / p-1}\right)-\mu_{2} \beta_{0} \gamma\left(\theta^{(1 / q-1)}\left(\frac{q-1}{q}\right) \phi_{q}^{q / q-1}\right)\right] \zeta \mathrm{d} x \\
& \leq \int_{\overline{\Omega_{\delta}}}\left[\lambda_{2} b(x) g(\underline{u})+\mu_{2} \beta(x) \gamma(\underline{v})\right] \zeta \mathrm{d} x .
\end{aligned}
$$


4

Complexity

On the contrary, on $\left(\Omega / \bar{\Omega}_{\delta}\right)$ we have

$$
\begin{aligned}
\theta \int_{\left(\Omega / \bar{\Omega}_{\delta}\right)}\left(\sigma_{p} \phi_{p}^{p}-\left|\nabla \phi_{p}\right|^{p}\right) \xi \mathrm{d} x & \leq \theta \sigma_{p} \int_{\left(\Omega / \bar{\Omega}_{\delta}\right)} \xi \mathrm{d} x \\
& =\theta \sigma_{p}\left(\frac{1}{d}+\frac{1}{d^{\prime}}\right) \int_{\left(\Omega / \bar{\Omega}_{\delta}\right)} \xi \mathrm{d} x \\
& \leq\left[\lambda_{1} a_{1} f\left(\theta^{(1 / q-1)} K_{2}\right)+\mu_{1} \alpha_{0} h\left(\theta^{(1 / p-1)} K_{1}\right)\right] \int_{\left(\Omega / \Omega_{\delta}\right)} \xi \mathrm{d} x \\
& \leq \int_{\left(\Omega / \bar{\Omega}_{\delta}\right)}\left[\lambda_{1} a(x) f(\underline{v})+\mu_{1} \alpha(x) h(\underline{u})\right] \xi \mathrm{d} x
\end{aligned}
$$

and similarly,

$$
\theta \int_{\left(\Omega / \bar{\Omega}_{\delta}\right)}\left(\sigma_{q} \phi_{q}^{q}-\left|\nabla \phi_{q}\right|^{q}\right) \zeta \mathrm{d} x \leq \int_{\left(\Omega / \Omega_{\delta}\right)}\left[\lambda_{2} b(x) g(\underline{u})+\mu_{2} \beta(x) \gamma(\underline{v})\right] \zeta \mathrm{d} x .
$$

Therefore, $(\underline{u}, \underline{v})$ is subsolution of problem (1).

Next, we construct a supersolution of (1). Let $\omega_{r}$ be a unique positive solution of

$$
\left\{\begin{array}{l}
-\triangle_{r} \omega_{r}=1 \text { in } \Omega, \\
\omega_{r}=0 \text { on } \partial \Omega
\end{array}\right.
$$

$$
\begin{aligned}
& \bar{u}=\frac{C}{v_{p}}\left(\frac{\lambda_{1}\|a\|_{\infty}+\mu_{1}\|\alpha\|_{\infty}}{1-v_{p}^{p-1}}\right)^{1 /(p-1)} \omega_{p}, \\
& \bar{v}=\left[\left(\frac{\lambda_{2}\|b\|_{\infty}+\mu_{2}\|\beta\|_{\infty}}{1-v_{q}^{q-1}}\right) g\left(C\left(\frac{\lambda_{1}\|a\|_{\infty}+\mu_{1}\|\alpha\|_{\infty}}{1-v_{p}^{p-1}}\right)^{1 /(p-1)}\right)^{1 /(q-1)}\right] \omega_{q},
\end{aligned}
$$

where $v_{r}=\left\|\omega_{r}\right\|_{\infty}, r=p, q$, and $C>0$ is a large number to be chosen later. We shall verify that $(\bar{u}, \bar{v})$ is a supersolution of (1) such that $(\bar{u}, \bar{v}) \geq(\underline{u}, \underline{v})$. By $(H 2)-(H 3)$, we can choose $C$ large enough so that

for $r=p, q$. We denote

$$
\begin{aligned}
\left(\frac{C}{\nu_{p}}\right)^{p-1} \geq & f\left(\left[\left(\frac{\lambda_{2}\|b\|_{\infty}+\mu_{2}\|\beta\|_{\infty}}{1-v_{q}^{q-1}}\right) g\left(C\left(\frac{\lambda_{1}\|a\|_{\infty}+\mu_{1}\|\alpha\|_{\infty}}{1-v_{p}^{p-1}}\right)^{(1 / p-1)}\right)^{(1 / q-1)}\right] \omega_{q}\right) \\
& +\mu_{1} h\left(\frac{\lambda_{1}\|a\|_{\infty}+\mu_{1}\|\alpha\|_{\infty}}{1-v_{p}^{p-1}}\right)^{(1 / p-1)} \omega_{p}
\end{aligned}
$$

Hence,

$$
\begin{array}{r}
\int_{\Omega}|\nabla \bar{u}|^{p-2} \nabla \bar{u} \cdot \nabla \xi \mathrm{d} x-\int_{\Omega}|\bar{u}|^{p-2} \bar{u} \cdot \xi \mathrm{d} x \\
=\left(\frac{C}{\nu_{p}}\right)^{p-1}\left(\lambda_{1}\|a\|_{\infty}+\mu_{1}\|\alpha\|_{\infty}\right) \int_{\Omega} \xi \mathrm{d} x .
\end{array}
$$

$$
\begin{aligned}
& \int_{\Omega}|\nabla \bar{u}|^{p-2} \nabla \bar{u} \cdot \nabla \xi \mathrm{d} x-\int_{\Omega}|\bar{u}|^{p-2} \bar{u} \cdot \xi \mathrm{d} x \\
& \geq \lambda_{1}\|a\|_{\infty} f\left(\left[\left(\frac{\lambda_{2}\|b\|_{\infty}+\mu_{2}\|\beta\|_{\infty}}{1-v_{q}^{q-1}}\right) g\left(C\left(\frac{\lambda_{1}\|a\|_{\infty}+\mu_{1}\|\alpha\|_{\infty}}{1-v_{p}^{p-1}}\right)^{1 /(p-1)}\right)^{1 /(q-1)}\right] \omega_{q}\right) \int_{\Omega} \xi \mathrm{d} x \\
& \quad+\mu_{1}\|\alpha\|_{\infty} \int_{\Omega} h\left(C\left(\frac{\lambda_{1}\|a\|_{\infty}+\mu_{1}\|\alpha\|_{\infty}}{1-v_{p}^{p-1}}\right)^{1 /(p-1)}\right) \xi \mathrm{d} x \\
& \geq \int_{\Omega}\left[\lambda_{1} a(x) f(\bar{v})+\mu_{1} \alpha(x) h(\bar{u})\right] \xi \mathrm{d} x .
\end{aligned}
$$


Next

$$
\begin{aligned}
& \int_{\Omega}|\nabla \bar{v}|^{q-2} \nabla \bar{v} \nabla \nabla \zeta \mathrm{d} x-\int_{\Omega}|\bar{v}|^{q-2} \bar{v} \cdot \zeta \mathrm{d} x \\
& =\left\{\left(\lambda_{2}\|b\|_{\infty}+\mu_{2}\|\beta\|_{\infty}\right) g\left(C\left(\frac{\lambda_{1}\|a\|_{\infty}+\mu_{1}\|\alpha\|_{\infty}}{1-\nu_{p}^{p-1}}\right)^{1 /(p-1)}\right)\right\} \omega_{q} \int_{\Omega} \xi \mathrm{d} x \\
& \geq\left[\begin{array}{l}
\lambda_{2}\|b\|_{\infty} g\left(C\left(\frac{\lambda_{1}\|a\|_{\infty}+\mu_{1}\|\alpha\|_{\infty}}{1-\nu_{p}^{p-1}}\right)^{1 /(p-1)}\right) \\
+\mu_{2}\|\beta\|_{\infty} g\left(C\left(\frac{\lambda_{1}\|a\|_{\infty}+\mu_{1}\|\| \|_{\infty}}{1-\nu_{p}^{p-1}}\right)^{1 /(p-1)}\right)
\end{array}\right] \int_{\Omega} \xi \mathrm{d} x .
\end{aligned}
$$

By (H3), choose $C$ large so that

$$
\begin{aligned}
& g\left(C\left(\frac{\lambda_{1}\|a\|_{\infty}+\mu_{1}\|\alpha\|_{\infty}}{1-v_{p}^{p-1}}\right)^{1 /(p-1)}\right) \\
& \quad \geq \gamma\left(\left[\left(\frac{\lambda_{2}\|b\|_{\infty}+\mu_{2}\|\beta\|_{\infty}}{1-v_{q}^{q-1}}\right) g\left(C\left(\frac{\lambda_{1}\|a\|_{\infty}+\mu_{1}\|\alpha\|_{\infty}}{1-v_{p}^{p-1}}\right)^{1 /(p-1)}\right)^{1 /(q-1)}\right]\left\|\omega_{q}\right\|_{\infty}\right) .
\end{aligned}
$$

Then, from (19), we have

$$
\begin{aligned}
& \int_{\Omega}|\nabla \bar{v}|^{q-2} \nabla \bar{v} \cdot \nabla \zeta \mathrm{d} x-\int_{\Omega}|\bar{v}|^{q-2} \bar{v} \cdot \zeta \mathrm{d} x \\
& \geq \lambda_{2}\|b\|_{\infty} g\left(C\left(\frac{\lambda_{1}\|a\|_{\infty}+\mu_{1}\|\alpha\|_{\infty}}{1-v_{p}^{p-1}}\right)^{1 /(p-1)}\right) \\
& \quad+\mu_{2}\|\beta\|_{\infty} \gamma\left(\left\{\left(\frac{\lambda_{2}\|b\|_{\infty}+\mu_{2}\|\beta\|_{\infty}}{1-v_{q}^{q-1}}\right) g\left(C\left(\frac{\lambda_{1}\|a\|_{\infty}+\mu_{1}\|\alpha\|_{\infty}}{1-v_{p}^{p-1}}\right)^{1 /(p-1)}\right)\right\}^{1 /(q-1)}\left\|\omega_{q}\right\|_{\infty}\right) \\
& \geq \int_{\Omega}\left[b(x) g(\bar{u})+\mu_{2} \beta(x) \gamma(\bar{v})\right] \zeta \mathrm{d} x .
\end{aligned}
$$

According to (19) and (20), we can conclude that $(\bar{u}, \bar{v})$ is a supersolution of (1). Furthermore, $\bar{u} \geq \underline{u}$ and $\bar{v} \geq v$ for $C$ large, Thus, there exists a solution $(u, v) \in W^{1, p}(\Omega) \cap$ $C(\bar{\Omega}) \times W^{1, q}(\Omega) \cap C(\bar{\Omega})$ of (1) with $\underline{u} \leq u \leq \bar{u}$ and $\underline{v} \leq v \leq \bar{v}$. This completes the Proof of Theorem 1 .

\section{Data Availability}

The data used to support the findings of this study are available from the corresponding author upon request.

\section{Conflicts of Interest}

The authors declare that there are no conflicts of interest regarding the publication of this manuscript.

\section{Authors' Contributions}

All authors contributed equally to this work. They have all read and approved the final manuscript.

\section{References}

[1] G. A. Afrouzi and K. J. Brown, "Positive solutions for a semilinear elliptic problem with sign-changing nonlinearity," Nonlinear Analysis: Theory, Methods \& Applications, vol. 36, no. 4, pp. 507-510, 1999.

[2] J. Ali and R. Shivaji, "Existence results for classes of Laplacian systems with sign-changing weight," Applied Mathematics Letters, vol. 20, no. 5, pp. 558-562, 2007. 
[3] S. Boulaaras, M. S. Touati Brahim, S. Bouzenada, and A. Zarai, "An asymptotic behavior and a posteriori error estimates for the generalized Schwartz method of advection-diffusion equation," Acta Mathematica Scientia, vol. 38, no. 4, pp. 1227-1244, 2018.

[4] N. Boumaaza and S. Boulaaras, "General decay for Kirchhoff type in viscoelasticity with not necessarily decreasing kernel," Mathematical Methods in the Applied Sciences, vol. 41, no. 16, pp. 6050-6069, 2018.

[5] N. Mezouar and S. Boulaaras, "Global existence of solutions to a viscoelastic non-degenerate Kirchhoff equation," Applicable Analysis, pp. 1-25, 2018, In press.

[6] Y. Bouizem, S. Boulaaras, and B. Djebbar, "Some existence results for an elliptic equation of Kirchhoff-type with changing sign data and a logarithmic nonlinearity," Mathematical Methods in the Applied Sciences, vol. 42, no. 7, pp. 2465-2474, 2019.

[7] D. D. Hai and R. Shivaji, "An existence result on positive solutions for a class of $p$-Laplacian systems," Nonlinear Analysis: Theory, Methods \& Applications, vol. 56, no. 7, pp. 1007-1010, 2004.

[8] N. Mezouar and S. Boulaaras, "Global existence and decay of solutions for a class of viscoelastic Kirchhoff equation," Bulletin of the Malaysian Mathematical Sciences Society, vol. 43, no. 1, pp. 725-755, 2020.

[9] S. H. Rasouli, Z. Halimi, and Z. Mashhadban, "A remark on the existence of positive weak solution for a class of -Laplacian nonlinear system with sign-changing weight," Nonlinear Analysis: Theory, Methods \& Applications, vol. 73, no. 2, pp. 385-389, 2010.

[10] S. Boulaaras and A. Allahem, "Existence of positive solutions of nonlocal $p(x)$-Kirchhoff evolutionary systems via sub-super solutions concept," Symmetry, vol. 11, no. 2, p. 253, 2019.

[11] G. A. Afrouzi, N. T. Chung, and S. Shakeri, "Existence of positive solutions for Kirchhoff type equations," Electronic Journal of Differential Equations, vol. 2013, pp. 1-8, 2013.

[12] S. Boulaaras and R. Guefaifia, "Existence of positive weak solutions for a class of Kirrchoff elliptic systems with multiple parameters," Mathematical Methods in the Applied Sciences, vol. 41, no. 13, pp. 5203-5210, 2018.

[13] Y. Chen, S. Levine, and M. Rao, "Variable exponent, linear growth functionals in image restoration," SIAM Journal on Applied Mathematics, vol. 66, no. 4, pp. 1383-1406, 2006.

[14] M. Ruzicka, Electrorheological Fluids: Modeling and Mathematical Theory, Springer-Verlag, Berlin, Germany, 2002.

[15] V. V. Zhikov, "Averaging of functionals of the calculus of variations and elasticity theory," Mathematics of the USSRIzvestiya, vol. 29, no. 1, pp. 33-66, 1987.

[16] G. A. Afrouzi, S. Shakeri, and N. T. Chung, "Existence of positive solutions for variable exponent elliptic systems with multiple parameters," Afrika Matematika, vol. 26, no. 1-2, pp. 159-168, 2015.

[17] S. Boulaaras, "A well-posedness and exponential decay of solutions for a coupled Lamé system with viscoelastic term and logarithmic source terms," Applicable Analysis, pp. 1-19, 2019, In press.

[18] S. Boulaaras, Y. Bouizem, and R. Guefaifia, "Existence of positive solutions of $(p(x), q(x))$-Laplacian parabolic systems with right hand side defined as a multiplication of two separate functions," Mathematical Methods in the Applied Sciences, vol. 43, no. 5, pp. 2615-2625, 2020.

[19] S. Boulaaras, "Some existence results for a new class of elliptic Kirchhoff equation with logarithmic source terms," Journal of
Intelligent \& Fuzzy Systems, vol. 37, no. 6, pp. 8335-8344, 2019, In press.

[20] Y. Bouizem, S. Boulaaras, and A. Ali, "Existence of positive solutions for a class of $(p(x), q(x))$-Laplacian elliptic systems with multiplication of two separate functions," Complexity, vol. 2020, Article ID 3756406, 10 pages, 2020.

[21] N. T. Chung, "Multiple solutions for a $p(x)$-Kirchhoff-type equation with sign-changing nonlinearities," Complex Variables and Elliptic Equations, vol. 58, no. 12, pp. 1637-1646, 2013.

[22] S. Boulaaras and N. Mezouar, "Global existence and decay of solutions of a singular nonlocal viscoelastic system with a nonlinear source term, nonlocal boundary condition, and localized damping term," Mathematical Methods in the Applied Sciences, 2020, In press.

[23] S. Boulaaras, "Existence of positive solutions of nonlocalp $(x)$ Kirchhoff hyperbolic systems via sub-super solutions concept," Journal of Intelligent \& Fuzzy Systems, pp. 1-13, 2020.

[24] R. Guefaifia and S. Boulaaras, "Sub-super solutions method for elliptic systems involving $\left(p_{1}, \ldots, p_{m}\right)$ Laplacian operator," Mathematical Methods in the Applied Sciences, vol. 43, no. 7, pp. 4191-4199, 2020.

[25] R. Guefaifia and S. Boulaaras, "Existence of positive solution for a class of $(p(x), q(x))$-Laplacian systems," Rendiconti del Circolo Matematico di Palermo Series 2, vol. 67, pp. 93-103, 2018.

[26] S. Boulaaras and D. Ouchenane, "General decay for a coupled Lamé system of nonlinear viscoelastic equations," Mathematical Methods in the Applied Sciences, vol. 43, no. 4, pp. 1717-1735, 2020.

[27] A. Menaceur, S. Boulaaras, R. Guefafia, and A. Alharbi, "Existence of positive weak solutions for quasilinear Kirchhoff elliptic systems via sub-super solutions concept," Mathematical Problems in Engineering, vol. 2020, Article ID 6527672, 6 pages, 2020.

[28] N. Mezouar, S. Boulaaras, and A. Alahem, "Global existence of solutions for the viscoelastic Kirchhoff equation with logarithmic source terms," Complexity, vol. 2020, Article ID 7105387, 25 pages, 2020.

[29] Q. H. Zhang, "Existence of positive solutions for a class of $p(x)$-Laplacian systems," Journal of Mathematical Analysis and Applications, vol. 333, pp. 591-603, 2007.

[30] F. Kamache, R. Guefaifia, S. Boulaaras, and A. Alharbi, "Existence of weak solutions for a new class of fractional $p$ Laplacian boundary value systems," Mathematics, vol. 8, no. 4, p. $475,2020$.

[31] X. L. Fan, "On the sub-supersolution method for $p(x)$-Laplacian equations," Journal of Mathematical Analysis and Applications, vol. 330, pp. 665-682, 2007.

[32] X. Fan and D. Zhao, "On the Spaces $L^{p(x)}(\Omega)$ and $W^{m, p(x)}(\Omega)$," Journal of Mathematical Analysis and Applications, vol. 263, no. 2, pp. 424-446, 2001. 